\title{
Effects of Rice Bran Extracts Fermented with Lactobacillus plantarum on Neuroprotection and Cognitive Improvement in a Rat Model of Ischemic Brain Injury
}

\author{
Jeong Hwa Hong ${ }^{1,8}$, Ji Yeong Kim ${ }^{2,}$, Seung Eun Baek ${ }^{2}$, Pajaree Ingkasupart ${ }^{1}$, \\ Hwa Jin Park ${ }^{3}$ and Sung Goo Kang ${ }^{2, \dagger}$ \\ ${ }^{I}$ Department of Smart Foods and Drugs, Inje University, Gyungnam 621-749, Korea \\ ${ }^{2}$ Department of Biological Sciences, Institute of Basic Science, Inje University, Gyungnam 621-749, Korea \\ ${ }^{3}$ Department of Biomedical Laboratory Science, Inje University, Gyungnam 621-749, Korea
}

\begin{abstract}
This work aimed to study whether rice bran extract fermented with Lactobacillus plantarum (LW) promotes functional recovery and reduces cognitive impairment after ischemic brain injury. Ischemic brain injury was induced by middle cerebral artery occlusion (MCAO) in rats. Four groups were studied, namely the (1) sham, (2) vehicle, (3) donepezil, and (4) LW groups. Animals were injected with LW once a day for 7 days after middle cerebral artery occlusion. LW group showed significantly improved neurological function as compared to the vehicle group, as well as enhanced learning and memory in the Morris water maze. The LW group showed the greatest functional recovery. Moreover, the LW group showed an enhanced more survival cells anti-apoptotic effect in the cortex and neural cell densities in the hippocampal DG and CA1. In addition, this group showed enhanced expression of neurotrophic factors, antioxidant genes, and the acetylcholine receptor gene, as well as synaptophysin (SYP), Fox-3 (NeuN), doublecortin (DCX), and choline acetyltransferase (ChAT) proteins. Our findings indicate that LW treatment showed the largest effects in functional recovery and cognitive improvement after ischemic brain injury through stimulation of the acetylcholine receptor, antioxidant genes, neurotrophic factors, and expression of NeuN, SYP, DCX, and ChAT.
\end{abstract}

Key Words: Rice bran, Lactobacillus plantarum, Fermentation, Cognition, Ischemia

\section{INTRODUCTION}

Brain ischemia is one of the cerebrovascular diseases resulted from thromboembolic occlusion of major intracerebral vessels, including the middle cerebral artery (Zhang et al., 2012). The result is a significant reduction in blood

* Received: April 22, 2015 / Revised: May 20, 2015

Accepted: May 28, 2015

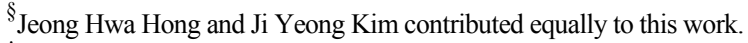

${ }^{\dagger}$ Corresponding author: Sung Goo Kang. Department of Biological Sciences, Institute of Basic Science, Inje University, 197, Inje-ro, Gimhae, Gyungnam 621-749, Korea.

Tel: +82-55-320-3213, Fax: +82-55-336-7706

e-mail: biosgkan@inje.ac.kr

@ The Korean Society for Biomedical Laboratory Sciences. All rights reserved. flow and energy failure critical for neural function and survival. Clinical studies report that memory damage and cognitive impairment are observed in cerebral ischemia (Nunn and Hodges, 1994; Kruyt et al., 2008; Kam et al., 2012). The cholinergic neurons play an important role in cognitive function and sensitive to cerebral ischemia (Block, 1999; Zhang et al., 2010).

Many neuroprotective drugs have been shown to ameliorate ischemic brain damage. The development of a neuroprotective drug evaluated a molecule targets such as glutamate excitotoxicity, free radical-mediated damage, or inflammatory mechanisms (De Keyser et al., 1999; Kaengkan et al., 2013). Some of the prospective targets include protein disulphide isomerase (PDI) (Descamps et al., 2009), and 
nuclear factor-E2-related factor 2 (Nrf2) (Kam et al., 2011). Several neurotrophic factors, such as brain-derived neurotrophic factor (BDNF), nerve growth factor (NGF), and glial cell line-derived neurotrophic factor (GDNF), play key roles in the differentiation and survival of neurons (Fantacci et al., 2013). The expression of the synaptic vesicle marker synaptophysin (SYP) is also modulated by neurotrophic factors (Gao et al., 2006). Doublecortin (DCX) is a microtubule-associated protein found in migrating neuronal precursor cells in the developing central nervous system (Choi et al., 2011). Choline acetyltransferase (ChAT) is involved in the synthesis of acetylcholine (ACh), and used as a marker for cholinergic neurons (Hu et al., 213). Middle cerebral artery occlusion (MCAO) results in the loss of cholinergic neurons via decreased ChAT and acetylcholineesterase activities (Wang et al., 2009).

Rice is an essential food and dietary ration worldwide, especially in Asian countries. It contains fiber and many types of antioxidants, such as phytic acid, ferulic acid and oryzanols (Soi-Ampornkul et al., 2012). Rice bran extract might have a potential nutraceutical for the prevention of oxidative stress in neurodegenerative diseases (Hagl et al., 2013; Baek et al., 2014). We found that fermentation of rice bran produced biologically active metabolites. Rice bran fermented with LW showed higher total phenolics content $(36.4 \mathrm{mg} / 100 \mathrm{~g})$ as compared to the unfermented rice bran $(24.0 \mathrm{mg} / 100 \mathrm{~g})$. Ferulic acid metabolite of rice bran was incread from $1.48 \mathrm{mg} / 100 \mathrm{~g}$ to $18.36 \mathrm{mg} / 100 \mathrm{~g}$.

This study aimed to determine whether rice bran extract fermented with Lactobacillus plantarum Hong (LW) promotes functional recovery from ischemic brain injury through the expression of genes encoding antioxidants, neurotrophic factors, SYP, ChAT, and DCX, and to evaluate the recovery of the ischemia-induced cognitive impairment.

\section{MATERIALS AND METHODS}

\section{Animals}

Male Sprague-Dawley rats weighing 280 320 g at the time of surgery were housed in group of two per cage at 22 $\pm 3^{\circ} \mathrm{C}$ under a $12 \mathrm{~h} / 12 \mathrm{~h}$ light dark cycle. They had free access to food and tap water. All experimental procedures were approved by the committee for animal experimentation and Institutional Animal Laboratory Review Board of the Inje University (2014-26).

\section{Fermentation and extraction of rice bran}

Fermentation and extraction of rice bran (LW) was performed according to the following procedures: For the starter culture, Lactobacillus plantarum Hong (KFCC 11556P) was cultured in MRS broth medium (Difco Laboratories, Detroit, MI, USA) at $37^{\circ} \mathrm{C}$ for $24 \mathrm{~h}$. Rice bran (100 g) was suspended in $900 \mathrm{~mL}$ of distilled water, followed by autoclaving at $121^{\circ} \mathrm{C}$ for $15 \mathrm{~min}$. After cooling the medium to room temperature, the starter was added at $5 \%$ of total volume and incubated at $37^{\circ} \mathrm{C}$ for $36 \mathrm{~h}$. The fermentation broth was autoclaved at $121^{\circ} \mathrm{C}$ for $15 \mathrm{~min}$ and centrifuged at $8,500 \times \mathrm{g}$ for $10 \mathrm{~min}$ to remove cell mass. The supernatant was filtrated and concentrated to 54 brix by vacuum evaporation.

\section{Experimental design for animals}

To investigate the neuroprotective and cognitive effects of LW on infarct volume, behavior, and gene expression changes, four groups were studied, namely the (1) sham (saline, $1 \mathrm{ml} / \mathrm{kg} ; \mathrm{n}=3$ ), (2) vehicle (saline, $1 \mathrm{ml} / \mathrm{kg} ; \mathrm{n}=3$ ), (3) positive (DPZ) group (Donepezil injection $1 \mathrm{mg} / \mathrm{kg} ; \mathrm{n}=$ 3), and (4) LW (LW injection $123 \mathrm{mg} / \mathrm{kg} ; \mathrm{n}=3$ ) groups. The animals were injected with DPZ or LW once a day for 6 days after MCAO. DPZ and LW were dissolved in saline and injected intraperitoneally.

\section{Induction of ischemic brain}

Cerebral ischemia was induced by MCAO according to the methods previously described with some modifications (Longa et al., 1989). After administration of anesthetics, the right common carotid artery was exposed. The external carotid artery was then ligated at the distal end and cut off. A 4-0 nylon thread precoated with silicon was inserted from the right external into the right internal carotid artery to a distance of $20 \mathrm{~mm}$ from the carotid bifurcation to occlude the origin of the right middle cerebral artery. After $1 \mathrm{~h}$, the thread was withdrawn for reperfusion. Rectal temperature was maintained at $37 \pm 0.5^{\circ} \mathrm{C}$ throughout the surgical 
procedure. The brains were removed, dissected into $2 \mathrm{~mm}$ thick coronal sections immersed sequentially in a $2 \%$ solution of 2,3,5-triphenyltetrazolium chloride (TTC) in normal saline at $37^{\circ} \mathrm{C}$ for $10 \mathrm{~min}$, and then fixed in $10 \%$ meutral buffered formalin for $10 \mathrm{~s}$. The infarct area in the brain section was measured by National Institutes of Health (NIH) Image software (Image J). The ischemic lesion volume was calculated as the sum of ischemic lesions from five brain slices from half of the hemisphere. All of the Functional tests rats were performed using a modified neurological severity score (mNSS) (Chen et al., 2001). The mNSS was a composite of sensory (visual, tactile, and proprioceptive), motor, reflex, and balance tests. Neurological function was graded from 0 to 18 (normal score, 0 ; maximal deficit score, 18).

\section{Analysis of transcriptional levels}

Brain sample of the lesioned side was harvested for RTPCR. Total RNA was extracted using the acid guanidinium isothiocyanate-phenol-chloroform method with TRI Reagent. The sequences of the primer pairs used were PDI: 5'-TCT GGA GGA GGA GGA CAA C-3', 5'-TGG AAA ACA CAT CGC TAT T-3'; Nrf2: 5'-AAG GAA GCT GGA AAA CAT TG-3', 5'-AAA TGG TGC CCA AGA AAT TA-3'; BDNF: 5'-GGT CAC AGC GGC AGA TAA AAA-3', 5'TTG GGT AGT TCG GCA TTG CGA-3'; NGF: 5'-ACA TCA AGG GCA AGG AGG TGA-3', 5'-TGA CAA AGG TGT GAG TCG TGG-3'; GDNF: 5'-GAC TTG GGT TTG GGC TAC GA-3', 5'-TGG TAA ACC AGG CTG TCG TC-3'; Acetylcholine receptor: 5'-GCC AAC CTC ACA AGA AGC TC-3', 5'-CCA GGA TGA AAA CCC AGA GA-3' and $\beta$-actin: 5'-CCA TCA TGA AGT GTG ACG TT-3', 5'-CCA CCA ATC CAC ACA GAG TA-3'. PCR products were separated by electrophoresis on a $1.5 \%$ agarose gel containing ethidium bromide. Band intensities were quantified by National Institutes of Health Image software (Image J) and normalized with $\beta$-actin.

\section{Analysis of survival cells}

Cell death detection was analyzed using terminal deoxynucleotidyl transferase (TdT)-catalyzed incorporation of fluorescein-labeled nucleotides to free the $3^{\prime}-\mathrm{OH}$ ends of
DNA in a template-independent manner using the In Situ Cell Death Detection Kit (Roche Molecular Biochemicals, Inc., Mannheim, Germany). Briefly, cortex and striatum samples were fixed with $4 \%$ paraformaldehyde in phosphate buffer (pH 7.4) and treated with $0.3 \% \mathrm{H}_{2} \mathrm{O}_{2}$ in methanol for $10 \mathrm{~min}$. After they were washed in PBS, and permeabilized in $0.1 \%$ Triton X-100 in $0.1 \%$ sodium citrate. After samples were incubated with the TdT enzyme in a humidified chamber for $60 \mathrm{~min}$, peroxidase-conjugated antibody was added for $30 \mathrm{~min}$ at $37^{\circ} \mathrm{C} \cdot 3,3^{\prime}$-diaminobenzidine (DAB) was incubated for $10 \mathrm{~min}$, followed by washing with PBS. The numbers of TUNEL-positive cells in the slide were counted in three randomly selected microscopic fields $200 \times$.

\section{Immunohistochemistry (IHC)}

After rice bran extracts treatment, rats were sacrificed and then fixed with $4 \%$ paraformaldehyde in $0.1 \mathrm{M}$ phosphate buffer by cardiac perfusion. Brains were embedded in O.C.T. compound, (Sakura Finetek USA, Inc.). Samples were sliced into coronal sections $(10 \mu \mathrm{m})$ using a cryostat (HM 525, Thermo Scientific, USA). Brain samples were blocked with normal serum for $2 \mathrm{~h}$ at room temperature, and incubated with primary antibodies for $24 \mathrm{~h}$ at $4{ }^{\circ} \mathrm{C}$. The primary antibodies were against NeuN (1:700, Chemicon, USA), $\beta$-Tubulin III (1:700, Sigma, USA), SYP (1:500, Millipore, USA), ChAT (1:100, Millipore, USA), and DCX (1:1,000, Abcam, England) diluted in tris-buffered saline (TBS) containing $1 \%$ bovine serum albumin (w/v) and $0.3 \%$ Triton-X 100. After rinsing in TBS, samples were incubated with secondary antibody-conjugated fluorescein isothiocyanate (FITC) (1:300, Sigma, USA) for $2 \mathrm{~h}$ at room temperature. Sections were then rinsed in TBS and mounted in an aqueous mounting medium. The signals were visualized by a fluorescence microscopy (Carl Zeiss Axioskop2+, Germany).

\section{Morris Water Maze test (MWM)}

Morris water maze test were tested for spatial learning and memory. A pool (180 cm in diameter $\times 55 \mathrm{~cm}$ deep) was filled with tap water $\left(23^{\circ} \mathrm{C}\right.$ at $35 \mathrm{~cm}$ deep $)$ and Nontoxic black paint was covered on the water surface. A platform (diameter, $10 \mathrm{~cm}$ ) was placed $1.5 \mathrm{~cm}$ below the surface of the water. The pool was divided into quadrants and the 


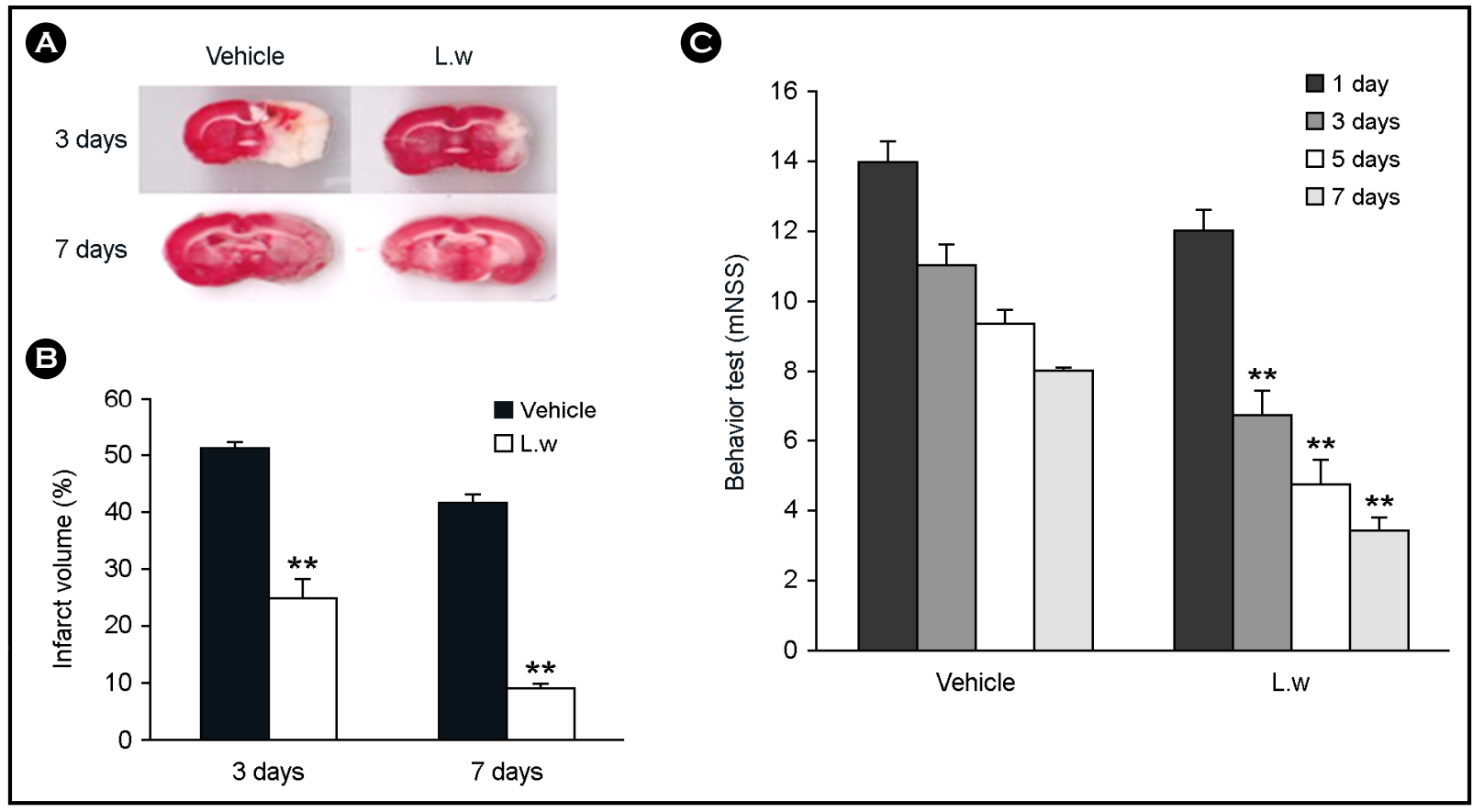

Fig. 1. Effect of LW on infarct volume and functional behavioral recovery at 3 and 7 days after MCAO. (A) TTC-stained coronal section from vehicle and LW group. (B) The infarct volume was calculated as the infarct areas $\mathrm{x}$ thickness $(2 \mathrm{~mm})$ and expressed as a percentage of the lesioned half of the brain. LW group shows a significantly lower infarct volume than the vehicle group 3 and 7 days after MCAO. (C) Neurological functional tests were performed at 1, 3,5 and 7 days after MCAO. LW group shows a significantly lower mNSS score than the vehicle group 3 days and 7 days after MCAO. Data are shown as means S.E.M. $(\mathrm{n}=3), * * P<0.01$ vs. individual vehicle group.

removable escape platform was placed in the center of one quadrant below the water level. The time for the animals to climb onto the hidden platform was recorded as escape latency. The test consisted of five trials each day for 4 consecutive days. In either case, the rat was left on the platform for $15 \mathrm{~s}$ before removal from the pool. A retention memory test was performed the next day. The platform was removed and the animals were placed into the water maze for $30 \mathrm{~s}$. The time taken for an animal to swim to the previous location of the platform was recorded. After each trial, the animal was dried with a towel before being returned to the cage (Morris, 1984).

\section{Novel Object Recognition test (NOR)}

NOR were carried out in an open field box $(80 \times 80 \times$ $40 \mathrm{~cm}$ ). Rats were habituated to the test box for $5 \mathrm{~min}$ without the presence of objects. After the habituation period, rats were placed into the test box with two identical objects and allowed to explore for $10 \mathrm{~min}$. Twenty-four hours after the training session, rats were again placed into the test box for 10 min with one familiar object and one novel object. The time that the animals spent exploring the novel and familiar objects was recorded. The animals were regarded to be exploring when they were facing, sniffing, or biting the object. The test box and objects were cleaned with $70 \%$ ethanol between sessions. Results were expressed as percentage of novel object recognition time (time percentage $\left.=t_{\text {novel }} /\left[t_{\text {novel }}+t_{\text {familiar }}\right] \times 100\right)($ Bevins and Besheer, 2006).

\section{Statistical analyses}

The significance of the differences in mean values of groups was determined using the student's $t$-tests. A significant difference was set at $P<0.05$. 


\section{RESULTS}

\section{LW treatment decreased infarct volume and increased functional recovery}

The total infarct volume was performed in sections between $4.00 \mathrm{~mm}$ and $-6.00 \mathrm{~mm}$ relative to the bregma, according to the rat brain atlas (Paxinos and Watson, 2006). The infarct volumes in the vehicle-treated group and the group treated with LW were $51.46 \pm 0.64$ and $24.45 \pm$ 3.33 of the total area of the brain section $3 \mathrm{~d}$ after MCAO, and were $41.58 \pm 1.26$ and $8.60 \pm 0.57$ of the total area of the brain section $7 \mathrm{~d}$ after MCAO, respectively. A significant decrease in the total infarct volume was also observed in the LW compared to the vehicle group $3 \mathrm{~d}$ and $7 \mathrm{~d}$ after MCAO $(P<0.05$ and $P<0.01$; Fig. 1 A and Fig. 1B). All treated groups showed no significant improvement $1 \mathrm{~d}$ after ischemia; however, 3, 5, and $7 \mathrm{~d}$ after treatment, there was significant functional recovery in the LW compared to the vehicle group $(P<0.05$ and $P<0.01$; Fig. 1C).

\section{LW treatment increased expression of genes encoding} antioxidants and neurotrophic factors

The effect of LW treatment on the expression of the genes encoding the antioxidant proteins PDI and Nrf2, and the genes encoding the endogenous neurotrophic factors BDNF, NGF, and GDNF was performed. At $3 \mathrm{~d}$ after focal cerebral ischemia, tissue extract from the lesioned hemisphere was subjected to RT-PCR analysis. The transcriptional levels of PDI and Nrf2 genes were significantly elevated in the LW group compared to the vehicle. The transcriptional levels of BDNF, NGF, and GDNF genes were also significantly elevated in the LW group compared to the vehicle group $(P$ $<0.05$ and $P<0.01$; Fig. 2A-D).

\section{Anti-apoptotic effects in the cortex, striatum, and SYP-} positive cells in the hippocampus

The analysis of TUNEL-positive cells showed that the LW group (118.2 \pm 21.16$)$ decreased in TUNEL-positive cells in the cortex compared to the vehicle group (202.2 \pm
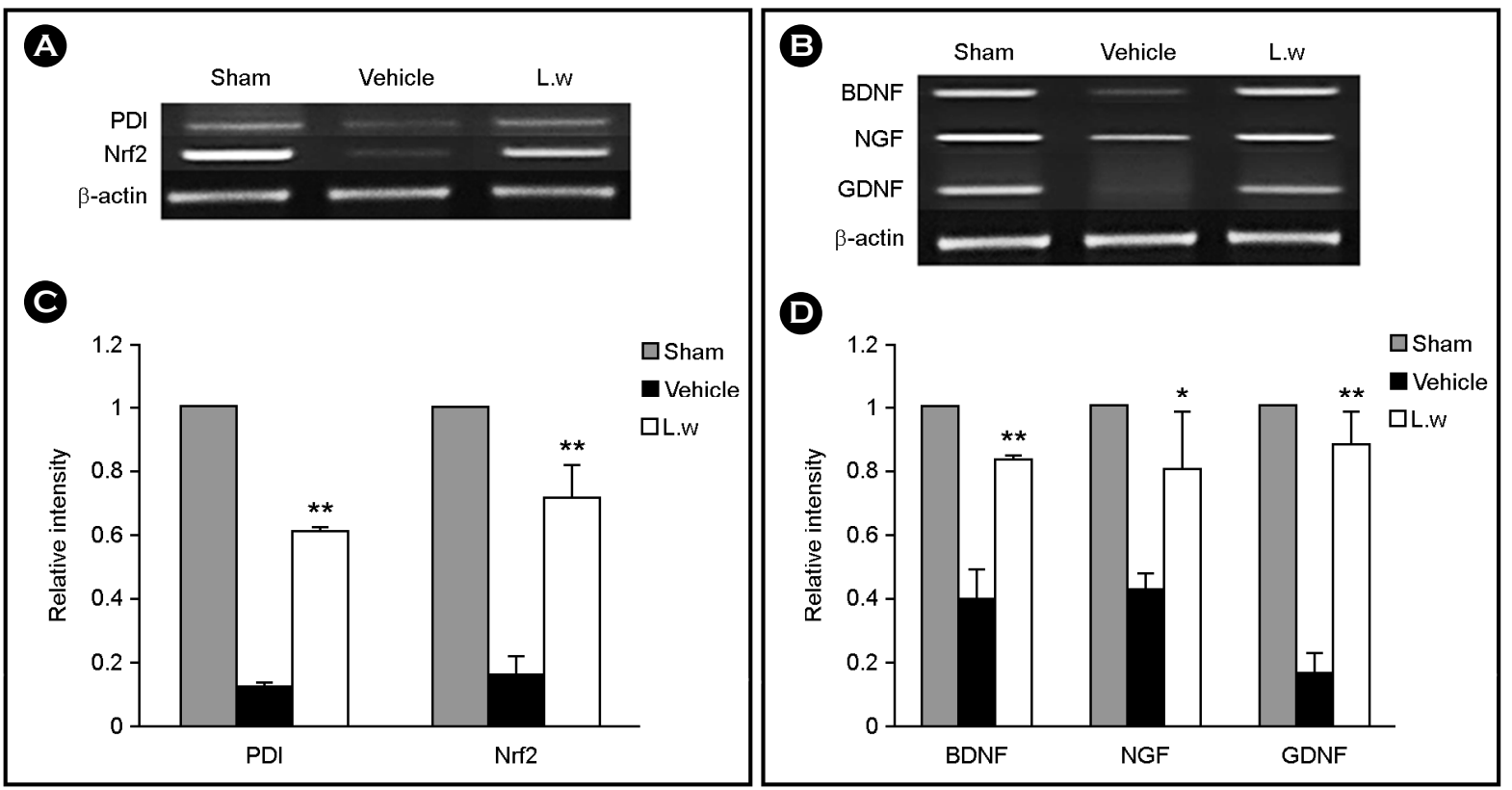

Fig. 2. Effect of LW on the gene expressions of PDI, Nrf2, BDNF, GDNF and NGF in the ipsilateral whole brain of the lesioned area. (A), (B) Specific gene expressions were determined in relation to the expression of $\beta$-actin using RT-PCR. (C), (D) The levels of PDI, Nrf2, BDNF, GDNF and NGF gene expressions were significantly increased in the LW treated group compared with the individual vehicle group. Experiments were repeated three times and individual values are expressed as the mean \pm S.E.M. $* P<0.05, * * P<0.01$ vs. individual vehicle group. 

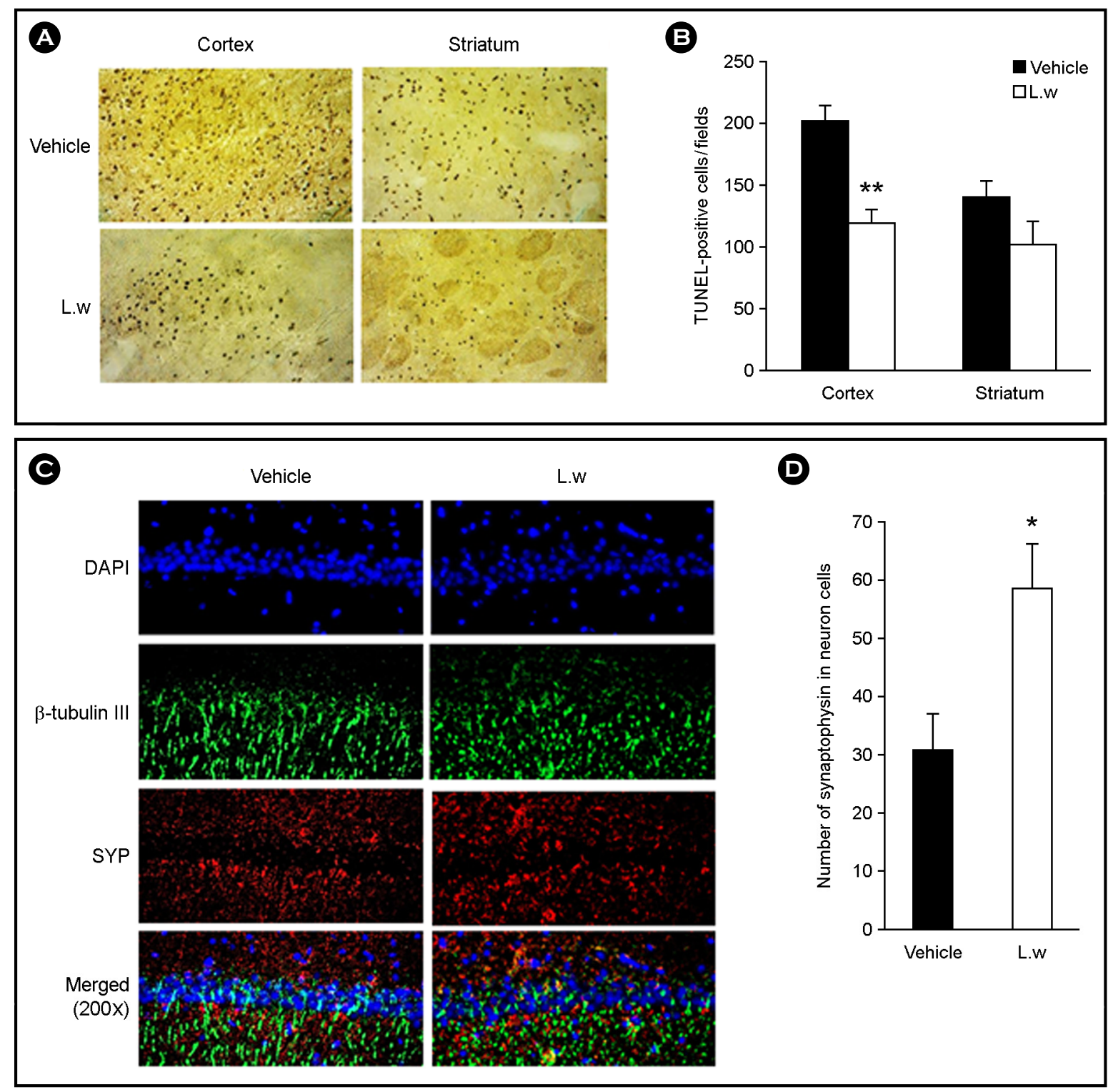

Fig. 3. Effect of LW on TUNEL staining and on the expression of SYP. (A) TUNEL staining in the cortex and striatum area of the ischemic lesioned side of vehicle and LW treatment $(200 \times)$. (B) Quantitative analysis of the number of TUNEL-positive cells. (C) IHC showed $\beta$-Tubulin III positive (green); a neuronal microtubule marker, SYP positive (red); a synaptophysin, synaptic vesicle marker and the double-labeling of $\beta$-Tubulin III (green), SYP (red) in the hippocampal CA1 region at 3 days after MCAO (200×). (D) SYP expression was significantly increased in the LW treated group compared with the individual vehicle group. Experiments were repeated three times and individual values are expressed as the mean \pm SEM. $* P<0.05, * * P<0.01$ vs. individual vehicle group.

11.33) $3 \mathrm{~d}$ after focal cerebral ischemia $(P<0.05$ and $P<$ 0.01 ; Fig. 3A and 3B). LW group (100.1 \pm 14.27$)$ had no anti-apoptotic effects compared to the vehicle group (139.7 $\pm 12.17) 3 \mathrm{~d}$ after focal cerebral ischemia (Fig. $3 \mathrm{~A}$ and 3B). The synaptic vesicle marker synaptophysin (SYP) and neuronal microtubule $\beta$-Tubulin III were evaluated in the hippocampal CA1 region of vehicle and treated animals. IHC showed that SYP expression in the LW group was significantly higher than in the vehicle group $(P<0.05$; Fig. $3 \mathrm{C}$ and Fig. 3D). 


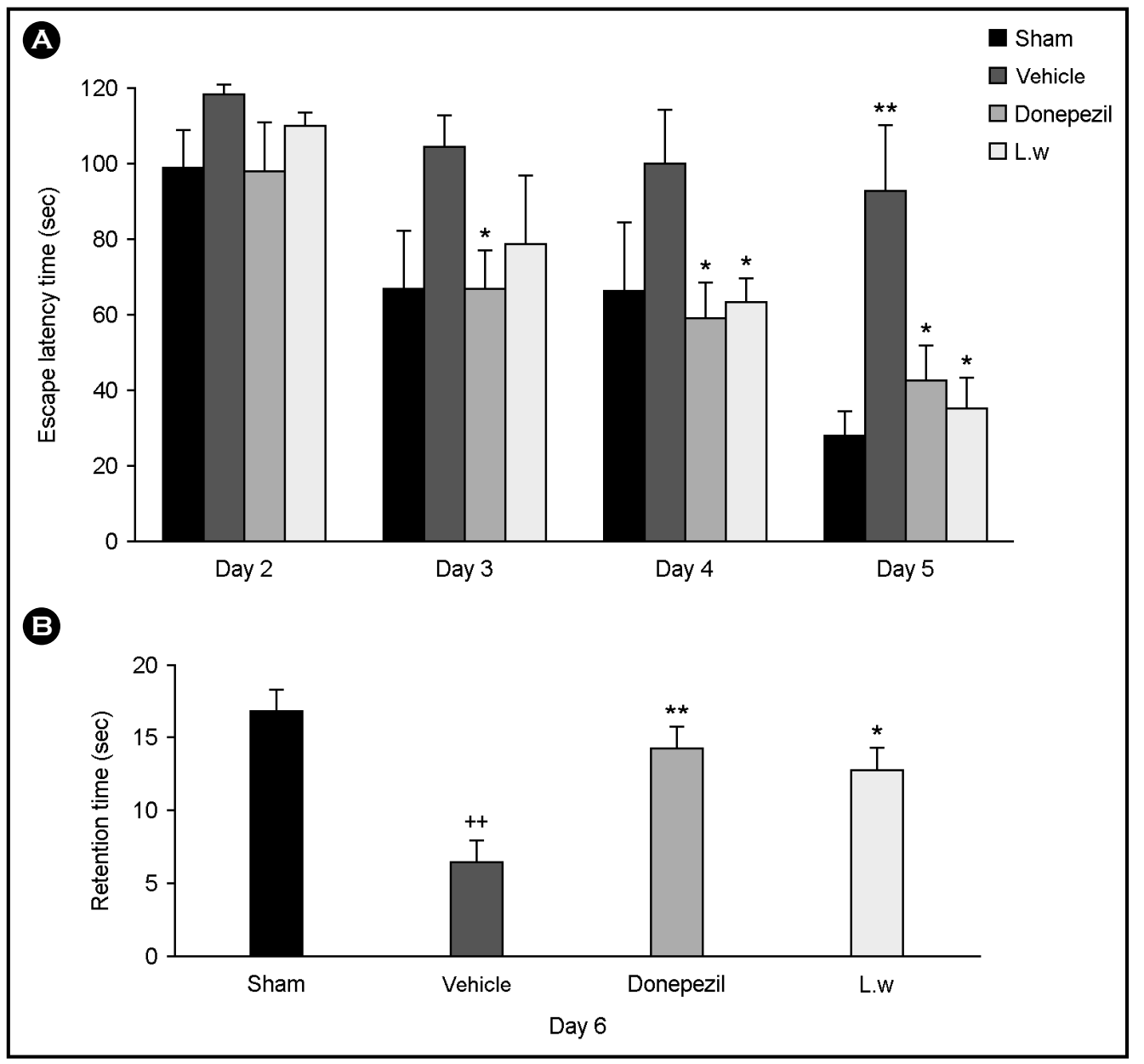

Fig. 4. Effect of LW on the ischemia-induced cognitive deficits in the Morris water maze. (A) Effect of LW on escape latency time in water maze. (B) Effect of LW on retention time in water maze on day 6 . The LW group shows a significantly longer retention time than the vehicle group. Data are shown as means $\operatorname{SEM}(\mathrm{n}=5) * P<0.05,{ }^{*} P<0.01$ vs. individual vehicle group; $++P<0.01$ vs. sham group.

\section{Cognitive function in MWM and NOR tests}

The effect of LW on learning and memory was determined using escape latency and retention time. The DPZ and LW groups showed significantly decreased escape latencies compared to the vehicle group. Five days after MCAO, the escape latency times in the sham, vehicle, DPZ, and LW groups were $27.88 \pm 6.40,92.44 \pm 16.94,42.28 \pm 9.11$, and $34.72 \pm 8.04$, respectively $(P<0.05$ and $P<0.01$; Fig. $4 \mathrm{~A})$. The vehicle group showed a significant decrease in retention time compared to the sham group. The DPZ and LW groups showed significantly increased retention times compared to the vehicle group. Six days after MCAO retention times in the sham, vehicle, DPZ, and LW groups were $16.8 \pm 1.36,6.4 \pm 1.44,14.2 \pm 1.43$, and $12.6 \pm$ 1.66, respectively $(P<0.05$ and $P<0.01$; Fig. 4B). Novel object recognition was performed to evaluate the effect of DPZ and LW on memory enhancement. The vehicle group spent a similar percentage of time exploring the novel and familiar objects. The DPZ and LW groups spent more time exploring the novel than the familiar object $7 \mathrm{~d}$ after MCAO. Novel object exploration times in the sham, vehicle, DPZ, and LW groups were $67.92 \pm 0.25,49.88 \pm 5.06,70.87$ \pm 2.41 , and $66.78 \pm 3.57$ at $7 \mathrm{~d}$ after $\mathrm{MCAO}(P<0.05$ and $P<0.01$; Fig. 5). 


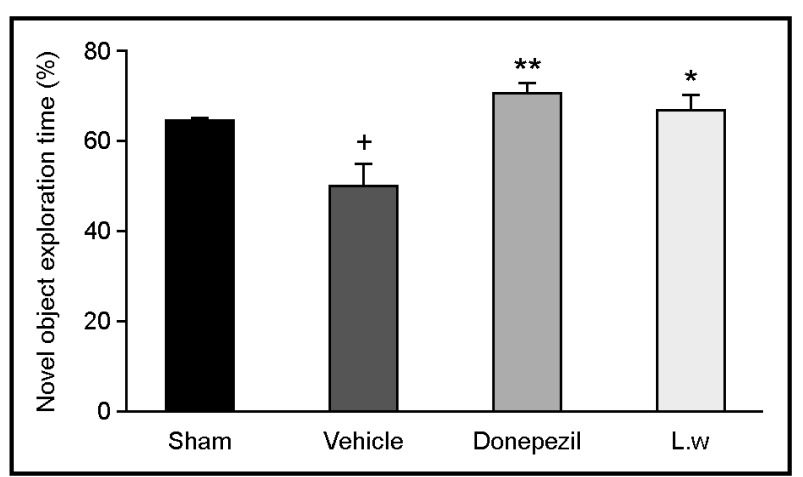

Fig. 5. Effect of $\mathbf{L W}$ on the ischemia-induced cognitive deficits in the novel object recognition. Novel object recognition test was performed at 7 days after MCAO. Data are shown as means SEM $(\mathrm{n}=5) * P<0.05,{ }^{* *} P<0.01$ vs. individual vehicle group; $+P<$ 0.05 vs. sham group.

\section{IHC of ChAT-positive cells in the striatum and DCX- expressing cells in the hippocampal DG}

The cholinergic neuron marker ChAT was evaluated in the striatum of vehicle and treated animals. The number of ChAT-immunoreactive neurons in the sham, vehicle, DPZ, LW groups was $25.00 \pm 1.00,12.33 \pm 2.96,23.00 \pm$ 1.53 , and $22.00 \pm 0.58$, respectively at $7 \mathrm{~d}$ after focal cerebral ischemia. IHC showed that ChAT positive cells in the striatum in the LW group was increased than that in the vehicle group $(P<0.05$ and $P<0.01$; Fig. $6 \mathrm{~A}$ and Fig. 6B). RT-PCR was performed to analyze the effects of LW on the expression levels of the acetylcholine receptor. The transcriptional level of the acetylcholine receptor gene was significantly elevated in the LW group compared to the vehicle $(P<0.05$ and $P<0.01$; Fig. $6 \mathrm{C}$ and Fig. $6 \mathrm{D})$ at $7 \mathrm{~d}$ after cerebral ischemia. The expression levels of neuronal markers NeuN and DCX were analysed in the hippocampal dentate gyrus (DG) of vehicle and LW group $7 \mathrm{~d}$ after MCAO. IHC showed that NeuN and DCX expression levels in the DG of the LW group were significantly higher than that in the vehicle group $(P<0.05$; Fig. $6 \mathrm{E}$ and Fig. $6 \mathrm{~F})$.

\section{DISCUSSION}

Rice bran has been noted as a neuroprotective agent because it contains antioxidants, such as oryzanols, phytic acid, tocopherols, and ferulic acid (Soi-Ampornkul et al., 2012). We previously reported that treatment with rice bran extracts resulted in functional recovery of the ischemic brain (Baek et al., 2014). Here we analyzed the effects of treatment with fermented rice bran extracts (LW) on the ischemic rat brain because lactic acid bacteria reportedly transformed ferulic acid 4-vinyl guaiacol, ehtylguaiacol, vanillin, vanillic acid, vinyl alcohol, and 4-ethyl phenol (Adamu et al., 2012; Kaur et al., 2013). This metabolite could play an important role in neural protection. There are no reports regarding these effects of LW. LW treatment decreased infarct sizes in the ischemic rat brain. The effect of LW on neural recovery may be related to the higher expression of neurotrophic factor genes and antioxidants genes. The antioxidant effect of LW may be related to the amelioration against ischemic-induced neurotoxicity, but the underlying mechanism is unknown. We performed RT-PCR analysis to quantify the expression of the antioxidant-related genes PDI and Nrf2. LW also increased the expression of the endogenous neurotrophic factor genes BDNF, NGF, and GDNF after ischemia. During brain development, neurotrophic factors are essential for neuronal survival and differentiation, as well as for the maintenance of neuronal functions (Snider, 1994). Accumulation of reactive oxygen species may have a bad effect on cell function and survival during oxidative stress, and trigger apoptotic cell death. Treatment of cells with many antioxidants suppresses apoptosis (Yu et al., 2005). LW extract could decrease DNA degradation and be at least partially associated with its anti-apoptotic actions by TUNEL assay in cortex and striatum after ischemia. Physiological as well as biochemical analyses using forebrain ischemia induced animal models have been demonstrated with CA1 pyramidal neurons (Nitatori et al., 1995). Our results of post-treatment with LW showed a significant high level of NeuN in the rat hippocampal DG after ischemia. Suggesting that there is a high density of neural cells in the DG region. In this study, immunostaining for endogenous SYP showed an increase in immunoreactivity in the hippocampal CA1 region of rats treated with the LW extract than in the CA1 of rats treated with vehicle. Our data show that treatment of the ischemic brain with the LW extract might enhance synaptic plasticity by 

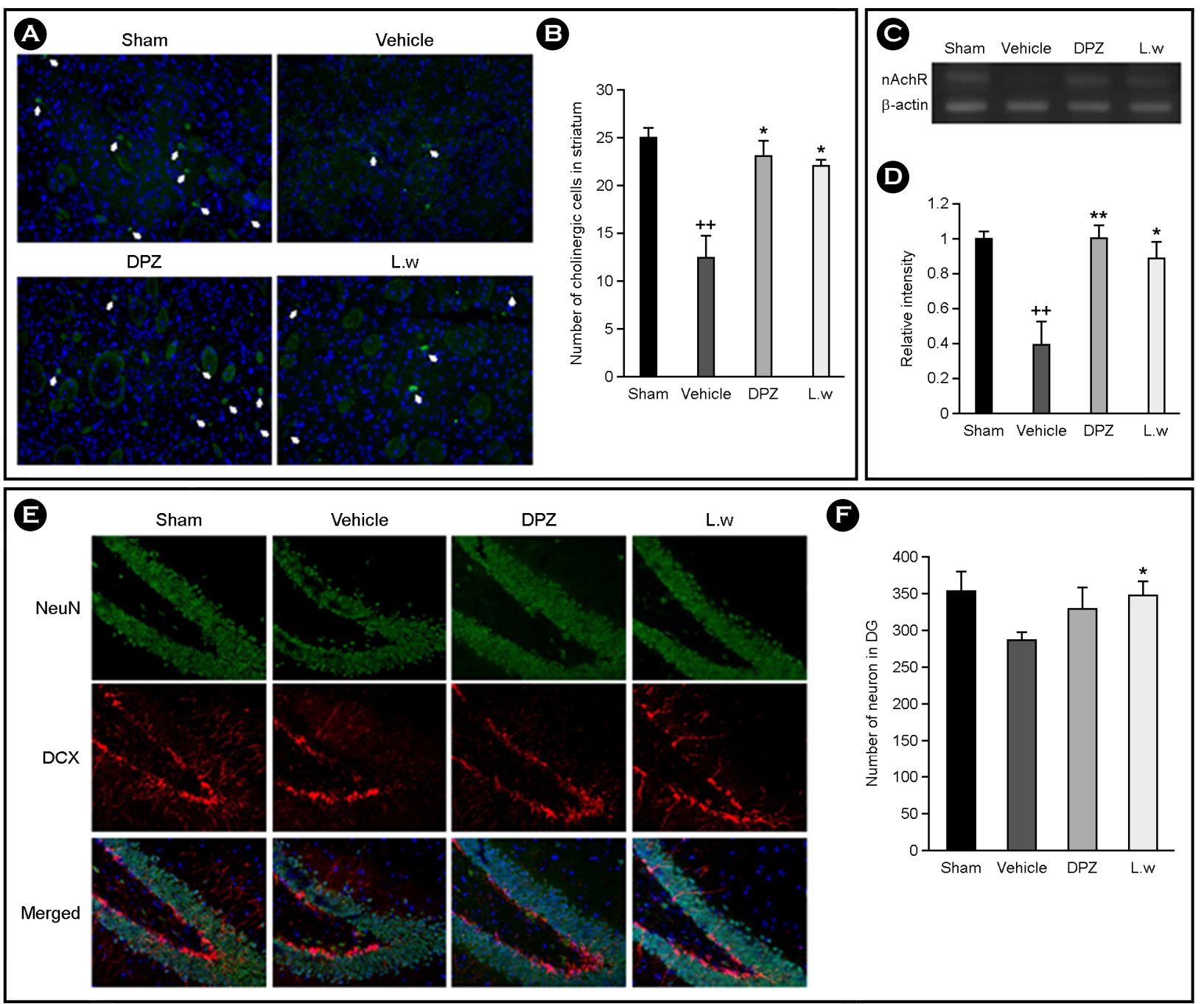

Fig. 6. Effect of LW on the expressions of ChAT, nAchR and DCX. (A) Immunohistochemistry showed ChAT positive neurons (green); a cholinergic neuron marker in the striatum at 7 days after MCAO $(200 \times)$. (B) Density of cholinergic cells expressing ChAT in the striatum region was significantly increased in the $\mathrm{LW}$ treated group compared with the individual vehicle group. (C), (D) The transcriptional level of acetylcholine receptor gene was significantly increased in the LW treated group compared with the individual vehicle group. (E) Immunohistochemistry showed DCX positive neurons (red); a neuronal marker in the hippocampal DG region at 7 days after MCAO (200×). (F) Number of neural cells expressed DCX was significantly increased in the LW treated group compared with the individual vehicle group. Data are the mean $\pm \operatorname{SEM}(\mathrm{n}=3) * P<0.05,{ }^{* *} P<0.01$ vs. individual vehicle group; $+P<0.01$ vs. sham group.

increasing endogenous rat SYP and neurotrophic factor expression. Synaptic plasticity is induced by a variety of neurotropic factors (Gao et al., 2006).

The Morris water maze (MWM) and novel object cognition (NOR) tasks are commonly used to test cognitive function. We utilized these behavioral paradigms to test cognitive function 2 to 7 days after MCAO. LW treatment enhanced learning and memory in the MWM and increased the preference ratio for novel objects in the NOR task. Donepezil, an acetylcholinesterase inhibitor, also ameliorated the spatial memory impairment in the MWM and NOR tests in our data. It was known that Donepezil increases extracellular ACh levels in the hippocampus in rats (Watanabe et al., 2008). Cerebral ischemia impaired spatial memory through cholinergic dysfunction, and the increase of ACh levels by donepezil may improve spatial memory 
impairment. The cholinergic system is hypothesized to play a pivotal role in learning and memory, which are improved by enhanced cholinergic neuron activity and suppression of AChE (Kaewkaen et al., 2012). Also it was known that ChAT activity was related with spatial memory impairment (Colombo and Gallagher, 1998). Treatment with LW increased cholinergic neurons and transcription of the acetylcholine receptor gene. It was known that DCX-positive cells correlates with neuronal cell proliferation and dendritic growth of new neurons that are generated in the DG of the rat hippocampus (Choi et al., 2011). Administration of LW increased the density of DCX-positive cells in the hippocampal DG region. The increase of cholinergic neurons and DCX expression by LW treatment may ameliorate ischemiainduced cognitive deficits.

This study demonstrates that LW treatment assists functional recovery and neuroprotection against ischemic brain injury through the stimulation of antioxidant gene and neurotrophic factor expression. Administration of LW could protect the brain from ischemic damage through an increase cholinergic neurons in a rat model of focal cerebral ischemia, and reduce cognitive impairment induced by focal cerebral ischemia. However, further studies are required to identify the active ingredient in the crude extract of fermented rice bran that is effective in ameliorating the negative outcomes of ischemia.

\section{Acknowledgements}

This research was supported by High Value-added Food Technology Development Program [112074-3], Ministry of Agriculture, Food and Rural Affairs.

\section{Conflict of interest}

The authors certify that they have NO affiliations with or involvement in any organization or entity with any financial interest, or non-financial interest in the subject matter or materials discussed in this manuscript.

\section{REFERENCES}

Adamu HA, Iqbal S, Chan KW, Ismali M. Biotransfromation of ferulic acid to 4-vinyl guaiacol by Lactobacillus farciminis.
2012. 11: $1177-1184$.

Baek SE, Kim JY, Song WT, Lee SH, Hong JH, Lee CK, Kang SG. Neuroprotective effect of rice bran extract supplement with ferulic acid in the rat model of ischemia brain injury. Anim Cells Syst. 2014. 18: 93-100.

Bevins RA, Besheer J. Object recognition in rats and mice: a onetrial non-matching-to-sample learning task to study 'recognition memory'. Nat Protoc. 2006. 1: 1306-1311.

Block F. Global ischemia and behavioural deficits. Prog Neurobiol. 1999. 58: 279-295.

Chen J, Sanberg PR, Li Y, Wang L, Lu M, Willing AE, SanchezRamos J, Chopp M. Intravenous administration of human umbilical cord blood reduces behavioral deficits after stroke in rats. Stroke. 2001. 32: 2682-2688.

Choi JG, Moon M, Jeong HU, Kim MC, Kim SY, Oh MS. Cistanches Herba enhances learning and memory by inducing nerve growth factor. Behav Brain Res. 2011. 216: 652-658.

Colombo PJ, Gallagher M. Individual differences in spatial memory and striatal ChAT activity among young and aged rats. Neurobiol Learn Mem. 1998. 70: 314-327.

De Keyser J, Sulter G, Luiten PG. Clinical trials with neuroprotective drugs in acute ischaemic stroke: are we doing the right thing? Trends Neurosci. 1999. 22: 535-540.

Descamps E, Petrault-Laprais M, Maurois P, Pages N, Bac P, Bordet R, Vamecq J. Experimental stroke protection induced by 4hydroxybenzyl alcohol is cancelled by bacitracin. Neurosci Res. 2009. 64: 137-142.

Fantacci C, Capozzi D, Ferrara P, Chiaretti A. Neuroprotective role of nerve growth factor in hypoxic-ischemic brain injury. Brain Sci. 2013. 3: 1013-1022.

Gao Y, Bezchlibnyk YB, Sun X, Wang JF, McEwen BS, Young LT. Effects of restraint stress on the expression of proteins involved in synaptic vesicle exocytosis in the hippocampus. Neuroscience. 2006. 141: 1139-1148.

Hagl S, Kocher A, Schiborr C, Eckert SH, Ciobanu I, Birringer M, El-Askary H, Helal A, Khayyal MT, Frank J, Muller WE, Eckert GP. Rice bran extract protects from mitochondrial dysfunction in guinea pig brains. Pharmacol Res. 2013. 76: 17 $-27$.

Hu X, Lu Y, Zhang Y, Li Y, Jiang L. Remote ischemic preconditioning improves spatial learning and memory ability after focal cerebral ischemia-reperfusion in rats. Perfusion. 2013. 28: 546-541.

Kaengkan P, Baek SE, Choi YW, Kam KY, Kim JY, Wu YR, Do 
BR, Kang SG. Combination effect of $p$-hydroxybenzyl alcohol and mesenchymal stem cells on the recovery of brain damage in a rat model of brain ischemia. Anim Cells and Sys. 2013. 17: 160-169.

Kaewkaen P, Tong-un T, Wattanathorn J, Muchimapura S, Kaewrueng W, Wongcharoenwanakit S. Mulberry fruit extract protects against memory impairment and hippocampal damage in animal model of vascular dementia. Evid Based Complement Alternat Med. 2012. Article ID 263520: 1-9.

Kam KY, Yu SJ, Jeong N, Hong JH, Jalin AM, Lee S, Choi YW, Lee CK, Kang SG. $p$-Hydroxybenzyl alcohol prevents brain injury and behavioral impairment by activating Nrf2, PDI, and neurotrophic factor genes in a rat model of brain ischemia. Mol Cells. 2011. 31: 209-215.

Kam KY, Jalin AM, Choi YW, Kaengkan P, Park SW, Kim YH, Kang SG. Ziprasidone attenuates brain injury after focal cerebral ischemia induced by middle cerebral artery occlusion in rats. Prog Neuropsychopharmacol Biol Psychiatry. 2012. 39: 69-74.

Kaur B, Chakraborty D, Kaur G, Kaur G. Biotranformation of rice bran to ferulic acid by Pediococcal Isolates. Appl Biochem Bitechnol. 2013. 170: 854-867.

Kruyt ND, Nys GM, van der Worp HB, van Zandvoort MJ, Kappelle LJ, Biessels GJ. Hyperglycemia and cognitive outcome after ischemic stroke. J Neurol Sci. 2008. 270: 141-147.

Longa EZ, Weinstein PR, Carlson S, Cummins R. Reversible middle cerebral artery occlusion without craniectomy in rats. Stroke. 1989. 20: 84-91.

Morris R. Developments of a water-maze procedure for studying spatial learning in the rat. J Neurosci Methods. 1984. 11: 47 -60 .

Nitatori T, Sato N, Waguri S, Karasawa Y, Araki H, Shibanai K, Kominami E, Uchiyama Y. Delayed neuronal death in the CA1 pyramidal cell layer of the gerbil hippocampus following transient ischemia is apoptosis. J Neurosci. 1995. 15: 1001
$-1011$.

Nunn J, Hodges H. Cognitive deficits induced by global cerebral ischaemia: relationship to brain damage and reversal by transplants. Behav Brain Res. 1994. 65: 1-31.

Paxinos G, Watson C. The rat brain in stereotaxic coordinates. 2006. Academic Press. Waltham, Massachusetts, USA, 2006.

Soi-Ampornkul R, Junnu S, Kanyok S, Liammongkolkul S, Katanyoo W, Umpornsirirat S. Antioxidative and neuroprotective activities of the pre-germinated brown rice extract. Food and Nutrition Sciences. 2012. 3: 135-140.

Snider WD. Functions of the neurotrophins during nervous system development: what the knockouts are teaching us. Cell. 1994. 77: 627-638

Wang J, Zhang HY, Tang XC. Cholinergic deficiency involved in vascular dementia: possible mechanism and strategy of treatment. Acta Pharm Sinic. 2009. 30: 879-888

Watanabe T, Iwasaki K, Ishikane S, Naitou T, Yoshimitsu Y, Yamagata N, Ozdemir MB, Takasaki K, Egashira N, Mishima K, Fujiwara M. Spatial memory impairment without apoptosis induced by the combination of beta-amyloid oligomers and cerebral ischemia is related to decreased acetylcholine release in rats. J Pharmacol Sci. 2008. 106: 84-91.

Yu SJ, Kim JR, Lee CK, Han JE, Lee JH, Kim HS, Hong JH, Kang SG. Gastrodia elata blume and an active component, $p$-hydroxybenzyl alcohol reduce focal ischemic brain injury through antioxidant related gene expressions. Biol Pharm Bull. 2005. 28: 1016-1020.

Zhang L, Fu F, Zhang X, Zhu M, Wang T, Fan H. Escin attenuates cognitive deficits and hippocampal injury after transient global cerebral ischemia in mice via regulating certain inflammatory genes. Neurochem Int. 2010. 57: 119-127.

Zhang R, Zhang H, Zhang Z, Niu J, Cui D, Xu S. Neuroprotective effects of pre-treament with 1-Carnitine and Acetyl-1-Carnitine on ischemic injury in vivo and in vitro. Int J Mol Sci. 2012 13: $2078-2090$ 